\title{
Ticagrelor monotherapy in patients with concomitant diabetes mellitus and chronic kidney disease: a post hoc analysis of the GLOBAL LEADERS trial
}

\author{
Chao Gao ${ }^{1,2,15 \dagger}$, Mariusz Tomaniak ${ }^{3,4 \dagger}$, Kuniaki Takahashi ${ }^{5}$, Hideyuki Kawashima ${ }^{5,15}$, Rutao Wang 1,2,15, \\ Hironori Hara ${ }^{5,15}$, Masafumi Ono ${ }^{5,15}$, Gilles Montalescot ${ }^{6}$, Scot Garg ${ }^{7}$, Michael Haude ${ }^{8}$, Ton Slagboom 9 , \\ Pascal Vranckx ${ }^{10}$, Marco Valgimigli ${ }^{11}$, Stephan Windecker ${ }^{11}$, Robert-Jan van Geuns ${ }^{2}$, Christian Hamm ${ }^{12}$, \\ Philippe Gabriel Steg ${ }^{13,14}$, Yoshinobu Onuma ${ }^{15}$, Dominick J. Angiolillo ${ }^{16}$ and Patrick W. Serruys ${ }^{15,17,18^{*}}$ (1)
}

\begin{abstract}
Background: Patients with both diabetes mellitus (DM) and chronic kidney disease (CKD) are a subpopulation characterized by ultrahigh ischemic and bleeding risk after percutaneous coronary intervention. There are limited data on the impact of ticagrelor monotherapy among these patients.

Methods: In this post hoc analysis of the GLOBAL-LEADERS trial, the treatment effects of the experimental (onemonth dual-antiplatelet therapy [DAPT] followed by 23-month ticagrelor monotherapy) versus the reference regimen (12-month DAPT followed by 12-month aspirin alone) were analyzed according to DM/CKD status. The primary endpoint was a composite endpoint of all-cause death or new Q-wave myocardial infarction at 2-years. The patientoriented composite endpoint (POCE) was defined as the composite of all-cause death, any stroke, site-reported MI and any revascularization, whereas net adverse clinical events (NACE) combined POCE with BARC type 3 or 5 bleeding events.
\end{abstract}

Results: At 2 years, the DM +/CKD + patients had significantly higher incidences of the primary endpoint (9.5\% versus 3.1\%, adjusted HR 2.16; 95\% Cl [1.66-2.80], $\mathrm{p}<0.001)$, BARC type 3 or 5 bleeding events, stroke, site-reported myocardial infraction, all revascularization, POCE, and NACE, compared with the DM-/CKD- patients. Among the $\mathrm{DM}+/$ CKD + patients, after adjustment, there were no significant differences in the primary endpoints between the experimental and reference regimen; however, the experimental regimen was associated with lower rates of POCE (20.6\% versus $\left.25.9 \%, \mathrm{HR} 0.74 ; 95 \% \mathrm{Cl}[0.55-0.99], \mathrm{p}=0.043, \mathrm{p}_{\text {interaction }}=0.155\right)$ and NACE $(22.7 \%$ versus $28.3 \%$, HR 0.75 ; $95 \% \mathrm{Cl}[0.56-0.99], p=0.044$, $p_{\text {interaction }}=0.310$ ), which was mainly driven by a lower rate of all revascularization, as compared with the reference regimen. The landmark analysis showed that while the experimental and reference regimen had similar rates of all the clinical endpoints during the first year, the experimental regimen was associated

\footnotetext{
*Correspondence: patrick.w.j.c.serruys@gmail.com

${ }^{\dagger}$ Chao Gao and Mariusz Tomaniak equally contributing as first authors

${ }^{18}$ Interventional Medicine and Innovation, National University of Ireland

Galway, P.O. University Road, Galway H91 TK33, Ireland

Full list of author information is available at the end of the article
}

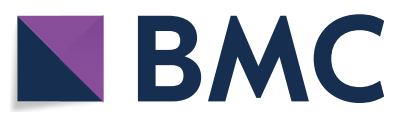

(c) The Author(s) 2020. This article is licensed under a Creative Commons Attribution 4.0 International License, which permits use, sharing, adaptation, distribution and reproduction in any medium or format, as long as you give appropriate credit to the original author(s) and the source, provide a link to the Creative Commons licence, and indicate if changes were made. The images or other third party material in this article are included in the article's Creative Commons licence, unless indicated otherwise in a credit line to the material. If material is not included in the article's Creative Commons licence and your intended use is not permitted by statutory regulation or exceeds the permitted use, you will need to obtain permission directly from the copyright holder. To view a copy of this licence, visit http://creativeco mmons.org/licenses/by/4.0/. The Creative Commons Public Domain Dedication waiver (http://creativecommons.org/publicdomain/ zero/1.0/) applies to the data made available in this article, unless otherwise stated in a credit line to the data. 
with significantly lower rates of POCE (5.8\% versus $\left.11.0 \%, \mathrm{HR} 0.49 ; 95 \% \mathrm{Cl}[0.29-0.82], \mathrm{p}=0.007, \mathrm{p}_{\text {interaction }}=0.040\right)$ and NACE (5.8\% versus $\left.11.2 \%, \mathrm{HR} 0.48 ; 95 \% \mathrm{Cl}[0.29-0.82], \mathrm{p}=0.007, \mathrm{p}_{\text {interaction }}=0.013\right)$ in the second year.

Conclusion: Among patients with both DM and CKD, ticagrelor monotherapy was not associated with lower rates of all-cause death or new Q-wave, or major bleeding complications; however, it was associated with lower rates of POCE and NACE. These findings should be interpreted as hypothesis-generating.

Clinical Trial Registration: ClinicalTrials.gov (NCT01813435).

Keywords: Chronic kidney disease, Diabetes mellitus, Percutaneous coronary intervention, DAPT, Ticagrelor, Aspirinfree antiplatelet strategies

\section{Background}

Patients with coronary artery disease (CAD) and concomitant diabetes mellitus (DM) or chronic kidney disease (CKD) are more susceptible to major adverse cardiovascular and cerebrovascular events [1]. Moreover, the presence of these risk factors is also associated with an increased risk of bleeding complications [2, 3]. DM and CKD frequently co-exist and given that DM is a wellestablished risk factor for renal dysfunction [2, 4], it is predicted that nearly $25 \%$ of DM patients have CKD [5].

Previously, a subgroup analysis of the PLATO study has demonstrated that in the acute coronary syndrome (ACS) population, those who had both DM and CKD were associated with a drastically unfavorable prognosis compared to those having one or neither of these comorbidities [6], and among the patients with both DM and CKD, the combination of ticagrelor with aspirin substantially reduced cardiovascular death, myocardial infarction (MI), or stroke compared with clopidogrel plus aspirin; however, the dual antiplatelet therapy (DAPT) with ticagrelor had a higher rate of TIMI non-CABGrelated major bleeding events.

In an attempt to mitigate bleeding risk while preserving the anti-ischemic efficacy, the "aspirin-free" antiplatelet strategy has been advocated [7-10]. The first and largest trial to date evaluating this concept -GLOBAL LEADERS, failed to show the superiority of ticagrelor monotherapy over standard DAPT in an all-comer patient population (in terms of all-cause mortality or new Q-wave MI) [7]. Nevertheless, understanding the impact of ticagrelor monotherapy after PCI in patients with DM and CKD in this large all-comer contemporary trial is still of clinical interest. The ever-growing prevalence of CKD in patients with DM [11, 12] underscores the need to specifically investigate the effects of different antiplatelet strategies in these ultrahigh risk patients.

On this background, here we report the results of a post hoc analysis of the GLOBAL LEADERS trial, in which we compared the outcomes of patients according to the presence or absence of DM and CKD, and also analyzed the effects of the experimental strategy (1-month DAPT followed by 23-month ticagrelor monotherapy) compared to the reference strategy (12-month DAPT followed by aspirin monotherapy for 12 months) after PCI in such defined subgroups.

\section{Methods}

The present study is a post hoc subgroup analysis of the GLOBAL LEADERS trial. GLOBAL LEADERS trial is a prospective, multi-center, randomized controlled trial (NCT01813435), which enrolled a total of 15,991 patients at 130 hospitals in 18 countries (Europe, Asia, Brazil, Australia and, Canada) between July 2013 and November 2015, and aimed to evaluate two antiplatelet strategies after PCI using bivalirudin and biolimus A9-eluting stents (Biomatrix) in an all-comers population [13]. Details of the study have been previously described. In brief, the experimental treatment strategy comprised aspirin 75-100 mg once daily in combination with ticagrelor $90 \mathrm{mg}$ twice daily for one month, followed by ticagrelor $90 \mathrm{mg}$ twice daily alone for 23 months (irrespective of clinical presentation). The reference treatment strategy included aspirin 75-100 mg daily in combination with either clopidogrel $75 \mathrm{mg}$ once daily in patients with stable CAD or ticagrelor $90 \mathrm{mg}$ twice daily in patients with ACS for 1 year, followed by aspirin 75-100 mg once daily alone for the following 12 months (from 12 to 24 months after PCI). Patients were followed up at 30 days and 3, $6,12,18$ and 24 months after the index procedure. An illustration of the antiplatelet strategy used in the trial is shown in Fig. 1.

The trial was approved by the institutional review board at each center and followed the ethical principles of the Declaration of Helsinki. All patients provided written informed consent prior to participation in the trial.

\section{Patients}

The GLOBAL LEADERS trial randomized 15,991 participants -23 patients withdrew consent and requested the deletion of their data from the database [7] -DM and CKD status was unavailable in 96 patients, leaving 15,872 patients $(99.2 \%)$ for the present analyses. Patients with DM or CKD were pre-specified subgroups of the GLOBAL LEADERS study [13]. However, the analyses 


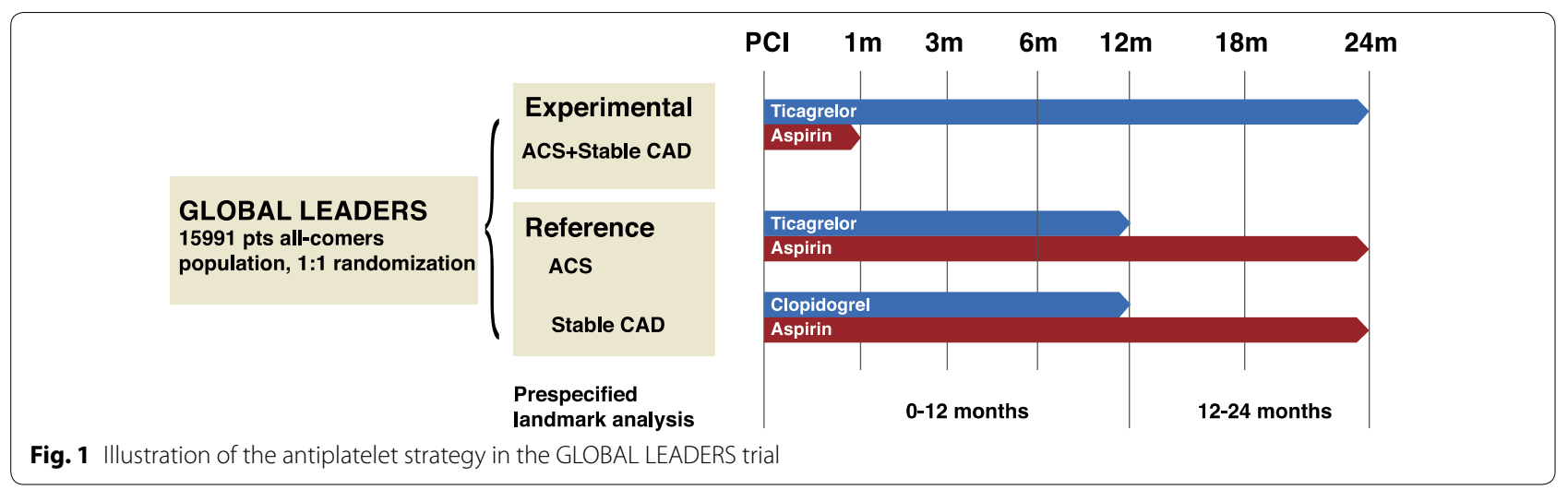

of current analyses were not pre-specified. As pre-specified in the trial protocol, CKD was defined at the time of randomization, using an eGFR cut-off of $60 \mathrm{ml} / \mathrm{min} / 1.73$ $\mathrm{m}^{2}$ (stage III to V CKD by KDIGO classification), calculated according to the Modification of Diet in Renal Disease (MDRD) equation [14]. A sensitivity analysis was performed by defining CKD using an eGFR cut-off of $90 \mathrm{ml} / \mathrm{min} / 1.73 \mathrm{~m}^{2}$ (equivalent to the stage II to $\mathrm{V}$ CKD by KDIGO classification, results shown in Additional file 1: Table S3). The status of DM was site-reported and defined at the time of randomization [13]. The PRECISEDAPT score was calculated by the online calculator [15].

\section{Outcomes}

The events definitions have been reported previously [16]. The primary endpoint was a composite of all-cause mortality or new Q-wave myocardial infarction (MI). The key secondary safety endpoint was investigator-reported Bleeding Academic Research Consortium (BARC) type 3 or 5 bleeding [17]. Other secondary endpoints included: individual components of the primary endpoint (allcause death, new Q-wave MI), individual components of key secondary safety endpoint (BARC defined bleeding type 3 or type 5 bleeding), any stroke, site-reported $\mathrm{MI}$, any revascularization, target vessel revascularization (TVR), definite stent thrombosis (ST) defined according to the Academic Research Consortium criteria [18]. The site-reported MI was defined according to the Third Universal Myocardial Infarction definition, as pre-specific in the study protocol [13]. The patient-oriented composite endpoint (POCE)-advocated by Academic Research Consortium (ARC)-2, and net adverse clinical events (NACE) were explored up to two years $[17,19]$. POCE was defined as the composite of all-cause death, any stroke, site-reported MI (including periprocedural or spontaneous with ST elevation MI [STEMI] or nonST-segment elevation MI [NSTEMI]) and any revascularization (re-PCI or coronary artery bypass graft surgery
[CABG] in the target or non-target vessel) [19], whereas NACE combined POCE with BARC type 3 or 5 bleeding events. Composite endpoints were analyzed hierarchically and the individual components of the composite endpoints were reported non-hierarchically.

\section{Statistical analysis}

All the analyses were performed by the intention-totreat principle. Continuous variables with normal distribution are expressed as mean \pm standard deviation and those with skewed distribution are expressed as median \pm interquartile range. Categorical variables are presented as counts and percentages. Means of 2 continuous variables were compared by independent samples Student's t-test or Mann-Whitney U test when appropriate. The frequencies of categorical variables were compared using Fisher's exact test. Survival was estimated by the Kaplan-Meier method. The impacts of CKD and $\mathrm{DM}$ on outcomes were assessed in the multivariable Cox proportional hazards model. The covariates in the multivariable model included age, sex, body mass index (BMI), clinical presentation (ACS versus stable CAD), stroke, peripheral vascular disease (PVD), chronic obstructive pulmonary disease (COPD), hypertension, previous PCI, hypercholesterolemia, current smoking status, treatment regimen (experimental versus. reference regimen), complex PCI, ACEI or ARB, beta-blockade, statin, Paris thrombotic risk score, and Paris bleeding risk score. A sensitivity analysis was conducted by adjusting the Cox proportional hazards model with the Propensity score (Propensity score was calculated by including all variables in Table 1). The detailed methods to calculate Propensity score and the results of the sensitivity analysis were shown in Additional file 1: Methods and Table S8. Cox proportionality assumptions were checked by using the Schoenfeld residuals against the transformed time and the assumptions were met in all models. Landmark analyses were performed at 365 days of follow-up, 
Table 1 Baseline Characteristics according to DM/CKD status

\begin{tabular}{|c|c|c|c|c|}
\hline Characteristic & $\begin{array}{l}\text { DM (-) CKD (-) } \\
n=10,513\end{array}$ & $\begin{array}{l}\text { DM (+) CKD (-) } \\
\mathrm{n}=3189\end{array}$ & $\begin{array}{l}\text { DM (-) CKD (+) } \\
n=1332\end{array}$ & $\begin{array}{l}\mathrm{DM}(+) \mathrm{CKD}(+) \\
\mathrm{n}=838\end{array}$ \\
\hline Age, years (SD) & $63.0(10.2)$ & $65.0(9.2)$ & $71.5(9.5)$ & $71.3(8.8)^{*}$ \\
\hline Male & $8387 / 10,513(79.8 \%)$ & $2445 / 3189(76.7 \%)$ & 830/1332 (62.3\%) & $518 / 838(61.8 \%)^{*}$ \\
\hline Mean body-mass index, kg/m² (SD) & $27.65(4.3)$ & $29.59(5.0)$ & $28.01(4.5)$ & $29.91(5.0)^{*}$ \\
\hline \multicolumn{5}{|l|}{ Medical history } \\
\hline Insulin-dependent diabetes mellitus & 0/10,513 (0.0\%) & $869 / 3155(27.3 \%)$ & 0/1332 (0.0\%) & $352 / 836(42.0 \%)$ \\
\hline Hypertension & $7047 / 10,471(67.3 \%)$ & $2721 / 3185$ (85.4\%) & $1118 / 1329(84.1 \%)$ & $770 / 838(91.9 \%)^{*}$ \\
\hline Hypercholesterolemia & $6771 / 10,196(64.4 \%)$ & 2421/3085 (75.9\%) & $879 / 1286(66.0 \%)$ & $634 / 813(75.7 \%)^{*}$ \\
\hline Current smoker & $3135 / 10,513(29.8 \%)$ & $686 / 3189(21.5 \%)$ & 207/1332 (15.5\%) & $110 / 838(13.1 \%)^{*}$ \\
\hline Previous stroke & $209 / 10,501(2.0 \%)$ & $120 / 3182(3.8 \%)$ & $48 / 1330(3.6 \%)$ & $44 / 838(5.3 \%)^{*}$ \\
\hline Previous peripheral vascular disease & $480 / 10,433(4.6 \%)$ & $273 / 3158(8.6 \%)$ & $121 / 1317(9.1 \%)$ & $126 / 826(15.0 \%)^{*}$ \\
\hline Chronic obstructive pulmonary disease & $485 / 10,474(4.6 \%)$ & $179 / 3174(5.6 \%)$ & $86 / 1327(6.5 \%)$ & $69 / 828(8.2 \%)^{*}$ \\
\hline Previous myocardial infarction & $2265 / 10,487(21.5 \%)$ & $815 / 3176(25.6 \%)$ & $344 / 1330(25.8 \%)$ & $269 / 835(32.1 \%)^{*}$ \\
\hline Previous $\mathrm{PCl}$ & $3107 / 10,504$ (29.6\%) & 1249/3186 (39.2\%) & 471/1331 (35.4\%) & $372 / 838(44.4 \%)^{*}$ \\
\hline Previous CABG & $477 / 10,506(4.5 \%)$ & 266/3185 (8.4\%) & $91 / 1331(6.8 \%)$ & $107 / 838(12.8 \%)^{*}$ \\
\hline Previous bleeding & $59 / 10,504(0.6 \%)$ & 18/3181 (0.6\%) & 15/1331 (1.1\%) & 6/838 (0.7\%) \\
\hline \multicolumn{5}{|l|}{ Clinical presentation } \\
\hline Stable coronary artery disease & $5298 / 10,513(50.4 \%)$ & $1913 / 3189$ (60.0\%) & $690 / 1332(51.8 \%)$ & $514 / 838(61.3 \%)$ \\
\hline Acute coronary syndrome & $5215 / 10,513(49.6 \%)$ & $1276 / 3189$ (40.0\%) & 642/1332 (48.2\%) & $324 / 838(38.7 \%)$ \\
\hline Complex PCl & 2976/10,513 (28.3\%) & $934 / 3189(29.3 \%)$ & $377 / 1332(28.3 \%)$ & 263/838 (31.4\%) \\
\hline Multivessel PCl & $2216 / 10,513(21.1 \%)$ & $671 / 3189(21.0 \%)$ & 282/1332 (21.2\%) & 189/838 (22.6\%) \\
\hline Lesion treated $\geq 3$ & $851 / 10,513(8.1 \%)$ & 266/3189 (8.3\%) & 113/1332 (8.5\%) & 68/838 (8.1\%) \\
\hline Stent implanted $\geq 3$ & $1793 / 10,513(17.1 \%)$ & $568 / 3189(17.8 \%)$ & 235/1332 (17.6\%) & $162 / 838(19.3 \%)$ \\
\hline Bifurcation $\mathrm{PCl}$ with $\geq 2$ stents & $323 / 10,513(3.1 \%)$ & $88 / 3189(2.8 \%)$ & $31 / 1332(2.3 \%)$ & 28/838 (3.3\%) \\
\hline Total stent length $>60 \mathrm{~mm}$ & $1346 / 10,513(12.8 \%)$ & 437/3189 (13.7\%) & 180/1332 (13.5\%) & $106 / 838(12.7 \%)$ \\
\hline Total Stent Length (SD) & $35.2(25.1)$ & $36.0(25.2)$ & $35.7(25.8)$ & $36.3(26.2)$ \\
\hline \multicolumn{5}{|l|}{ Medications on discharge } \\
\hline ACE-inhibition and/or ARB & $6346 / 10,450(60.4 \%)$ & 1986/3162 (62.3\%) & 730/1320 (54.8\%) & $457 / 826(54.5 \%)$ \\
\hline Beta-blockade & $8194 / 10,452(77.9 \%)$ & 2577/3163 (80.8\%) & 1069/1321 (80.3\%) & $669 / 826(79.8 \%)$ \\
\hline Statin & $9718 / 10,459(92.4 \%)$ & 2916/3168 (91.4\%) & $1212 / 1322(91.0 \%)$ & $764 / 827(91.2 \%)$ \\
\hline Paris bleeding risk score [31] (IQR) & $3(2,4)$ & $3(2,4)$ & $6(5,7)$ & $6(5,7)^{*}$ \\
\hline Paris thrombotic risk score (IQR) & $2(0,4)$ & $3(2,4)$ & $4(2,7)$ & $5(4,7)^{*}$ \\
\hline Paris bleeding risk score $\geq 8$ & $100 / 10,039(1.0 \%)$ & $41 / 3060(1.3 \%)$ & 269/1288 (20.9\%) & $189 / 803(23.5 \%)^{*}$ \\
\hline Paris thrombotic risk score $\geq 5$ & $140 / 10,506(1.3 \%)$ & $655 / 3185(20.8 \%)$ & 243/1331 (18.3\%) & $615 / 838(73.4 \%)^{*}$ \\
\hline PRECISE DAPT score [15] (IQR) & $14(9,19)$ & $15(10,20)$ & $27(23,32)$ & $29(24,34)^{*}$ \\
\hline PRECISE DAPT score $\geq 25$ & $731 / 9849(7.4 \%)$ & $323 / 3007(10.7 \%)$ & $846 / 1266(66.8 \%)$ & $567 / 799(71.0 \%)^{*}$ \\
\hline \multicolumn{5}{|l|}{ Antiplatelet therapy } \\
\hline Reference treatment strategy & $5297 / 10,513(50.4 \%)$ & 1575/3189 (49.4\%) & $662 / 1332(49.7 \%)$ & $410 / 838(48.9 \%)$ \\
\hline Experimental treatment strategy & $5216 / 10,513(49.6 \%)$ & $1614 / 3189(50.6 \%)$ & $670 / 1332(50.3 \%)$ & $428 / 838(51.1 \%)$ \\
\hline
\end{tabular}

Data are $\mathrm{n} / \mathrm{N}(\%)$, unless otherwise specified. Denominators vary because medical history data were incomplete

* The comparison between DM-/CKD + and DM +/CKD + was statistically significant

which was based on the prespecified landmark point in the GLOBAL LEADERS design. So far, there have been 24 subgroup analyses of the GLOBAL LEADERS trial. However, taking into account the post hoc and exploratory nature of the analysis, there was no formal correction for multiple testing [20]. Analyses were performed using R-project ( $\mathrm{R}$ Foundation, Vienna, Austria). A two-sided $p$ value less than 0.05 was considered as statistical significance.

\section{Results}

Patients and outcomes according to DM and CKD status A total of 15,872 patients from the GLOBAL LEADERS trial population were classified according to the DM and 
CKD status as follows: DM-/CKD- $(\mathrm{n}=10,513), \mathrm{DM}+/$ CKD- $(\mathrm{n}=3189), \mathrm{DM}-/ \mathrm{CKD}+(\mathrm{n}=1332)$, and $\mathrm{DM}+/$ $\mathrm{CKD}+(\mathrm{n}=838)$. Baseline characteristics are presented in Table 1. Patients with DM+/CKD + were older, more often had a prior history of revascularization (PCI or CABG), previous stroke, previous $\mathrm{MI}$, PVD, COPD. In $\mathrm{DM}+/ \mathrm{CKD}+$ patients, the percentages of patients who had a Paris bleeding risk score $\geq 8$ (23.5\%), thrombotic risk score $\geq 5$ (73.4\%), and PRECISE-DAPT score $\geq 25$ (71.0\%) were higher compared with DM-/CKD- patients.

The DM+/CKD + patients had a 2.16-fold higher incidence of the primary endpoint at 24 months, compared with the DM-/CKD- individuals (9.5\% versus $3.1 \%$, adjusted HR 2.16; 95\% CI [1.66-2.80], Table 2). The DM-/ CKD + (6.9\%, adjusted HR 1.53; 95\% CI [1.20-2.80]) and DM+/CKD- patients (4.6\%, adjusted HR 1.40; $95 \%$ CI [1.15-1.72]) had intermediate risk profile. With the
$\mathrm{DM}+/ \mathrm{CKD}+$ patients exhibiting the highest risk, the hazard ratio gradually decreased in the order of DM-/ $\mathrm{CKD}+, \mathrm{DM}+/ \mathrm{CKD}-$ and DM-/CKD- $\left(\mathrm{P}_{\text {Trend }}<0.001\right.$; Fig. 2 and Table 2). Similar trends were observed in the key secondary endpoint (Bleeding Academic Research Consortium [BARC] type 3 or 5 bleeding), and other secondary endpoints including all-cause mortality, stroke, MI, revascularization, TVR, POCE, and NACE (Table 2).

\section{Outcomes of experimental versus reference regimen according to CKD and DM status}

Compared with the reference regimen (DAPT for 12 months and then aspirin for 12 months), the experimental regimen (DAPT for 1 month followed by ticagrelor monotherapy for 23 months) did not show lower rates of the primary or the key safety secondary endpoints in $\mathrm{DM}+/ \mathrm{CKD}+$ patients, or in any of the other three

Table 2 Clinical outcomes according to DM/CKD subgroup

\begin{tabular}{|c|c|c|c|c|c|c|c|c|}
\hline & \multicolumn{2}{|c|}{ DM (-) CKD (-) } & \multicolumn{2}{|l|}{$\mathrm{DM}(+)$ CKD (-) } & \multicolumn{2}{|l|}{$\mathrm{DM}(-) \mathrm{CKD}(+)$} & \multirow{2}{*}{$\begin{array}{l}\mathrm{DM}(+) \mathrm{CKD}(+) \\
\mathrm{HR}(95 \% \mathrm{Cl})\end{array}$} & \multirow[t]{2}{*}{$P_{\text {trend }}$} \\
\hline & $n=10,513$ & $\mathrm{HR}(95 \% \mathrm{Cl})$ & $\mathrm{HR}(95 \% \mathrm{Cl})$ & $n=1332$ & $\mathrm{HR}(95 \% \mathrm{Cl})$ & $n=838$ & & \\
\hline $\begin{array}{l}\text { All-cause mortality or New } \\
\text { Q-wave Ml }\end{array}$ & $330(3.1 \%)$ & 1.00 (Ref) & $1.32(1.07-1.61)$ & $92(6.9 \%)$ & $1.54(1.21-2.49)$ & $80(9.5 \%)$ & $1.91(1.47-2.49)$ & $<0.001$ \\
\hline All-cause mortality & $226(2.1 \%)$ & 1.00 (Ref) & $1.37(1.08-1.75)$ & $74(5.6 \%)$ & $1.67(1.27-2.81)$ & $67(8 \%)$ & $2.09(1.55-2.81)$ & $<0.001$ \\
\hline New Q-wave Ml & $108(1 \%)$ & 1.00 (Ref) & $1.29(0.9-1.86)$ & $19(1.4 \%)$ & $1.19(0.72-2.3)$ & $13(1.6 \%)$ & $1.26(0.69-2.3)$ & 0.257 \\
\hline Stroke & $78(0.7 \%)$ & 1.00 (Ref) & $1.86(1.28-2.71)$ & $18(1.4 \%)$ & $1.17(0.68-3.48)$ & $19(2.3 \%)$ & $2.04(1.19-3.48)$ & 0.013 \\
\hline $\mathrm{Ml}$ & $273(2.6 \%)$ & 1.00 (Ref) & $1.48(1.18-1.85)$ & $52(3.9 \%)$ & $1.61(1.18-3.49)$ & $53(6.3 \%)$ & $2.54(1.85-3.49)$ & 0.001 \\
\hline Any Revascularization & 917 (8.7\%) & 1.00 (Ref) & $1.26(1.11-1.43)$ & $129(9.7 \%)$ & $1.17(0.96-1.91)$ & $113(13.5 \%)$ & $1.55(1.26-1.91)$ & $<0.001$ \\
\hline TVR & $466(4.4 \%)$ & 1.00 (Ref) & $1.5(1.27-1.78)$ & $71(5.3 \%)$ & $1.28(0.99-2.37)$ & $67(8.0 \%)$ & $1.8(1.38-2.37)$ & $<0.001$ \\
\hline Definite stent thrombosis & $82(0.8 \%)$ & 1.00 (Ref) & $1.08(0.69-1.7)$ & $13(1.0 \%)$ & $1.43(0.78-2.23)$ & $6(0.7 \%)$ & $0.94(0.4-2.23)$ & 0.569 \\
\hline MACE & $394(3.7 \%)$ & 1.00 (Ref) & $1.43(1.19-1.71)$ & $101(7.6 \%)$ & $1.4(1.11-2.42)$ & $93(11.1 \%)$ & $1.9(1.49-2.42)$ & $<0.001$ \\
\hline POCE & $1242(11.8 \%)$ & 1.00 (Ref) & $1.3(1.17-1.45)$ & $219(16.4 \%)$ & $1.3(1.12-2.06)$ & $194(23.2 \%)$ & $1.75(1.49-2.06)$ & $<0.001$ \\
\hline NACE & $1360(12.9 \%)$ & 1.00 (Ref) & $1.27(1.15-1.41)$ & 245 (18.4\%) & $1.29(1.12-2.03)$ & $213(25.4 \%)$ & $1.74(1.49-2.03)$ & $<0.001$ \\
\hline BARC 3 or 5 bleeding & $188(1.8 \%)$ & 1.00 (Ref) & $1.05(0.78-1.41)$ & $44(3.3 \%)$ & $1.18(0.83-2.39)$ & $37(4.4 \%)$ & $1.64(1.12-2.39)$ & 0.02 \\
\hline BARC 5 bleeding & $27(0.3 \%)$ & 1.00 (Ref) & $0.51(0.2-1.3)$ & $8(0.6 \%)$ & $1.16(0.5-2.09)$ & $5(0.6 \%)$ & $0.71(0.24-2.09)$ & 0.704 \\
\hline BARC 3 bleeding & $173(1.6 \%)$ & 1.00 (Ref) & $1.12(0.83-1.52)$ & $41(3.1 \%)$ & $1.22(0.85-2.71)$ & $35(4.2 \%)$ & $1.84(1.25-2.71)$ & 0.005 \\
\hline BARC 3a bleeding & 77 (0.7\%) & 1.00 (Ref) & $1.31(0.85-2.01)$ & $25(1.9 \%)$ & $1.64(1.02-2.63)$ & $13(1.6 \%)$ & $1.42(0.76-2.63)$ & 0.054 \\
\hline BARC 3b bleeding & $74(0.7 \%)$ & 1.00 (Ref) & $1.01(0.62-1.64)$ & $17(1.3 \%)$ & $1.29(0.73-3.71)$ & $14(1.7 \%)$ & $2.02(1.1-3.71)$ & 0.043 \\
\hline BARC 3 c bleeding & $38(0.4 \%)$ & 1.00 (Ref) & $0.78(0.37-1.65)$ & $3(0.2 \%)$ & $0.38(0.11-4.56)$ & $10(1.2 \%)$ & $2.11(0.98-4.56)$ & 0.524 \\
\hline BARC 2 bleeding & $489(4.7 \%)$ & 1.00 (Ref) & $1(0.83-1.21)$ & $82(6.2 \%)$ & $1.07(0.84-1.52)$ & $54(6.4 \%)$ & $1.14(0.85-1.52)$ & 0.388 \\
\hline BARC 2, 3 or 5 bleeding & $647(6.2 \%)$ & 1.00 (Ref) & $1.01(0.86-1.19)$ & $118(8.9 \%)$ & $1.11(0.9-1.62)$ & $84(10 \%)$ & $1.27(1-1.62)$ & 0.063 \\
\hline
\end{tabular}

Data are $\mathrm{n} / \mathrm{N}(\%)$, unless otherwise specified

$\mathrm{MI}$, Myocardial ischemia

TVR, Target vessel revascularization

MACE, all-cause death, any stroke, or non-fatal new Q-wave MI

POCE, all-cause death, any stroke, any myocardial infarction or any revascularization

BARC, Bleeding Academic Research Consortium

NACE, POCE and BARC 3 or 5 bleeding

Adjusted to age, sex, body mass index (BMI), clinical presentation (ACS versus stable CAD), stroke, peripheral vascular disease (PVD), chronic obstructive pulmonary disease (COPD), previous $\mathrm{PCl}$, hypercholesterolemia, hypertension, current smoking status, treatment regimen (experimental versus. reference regimen), complex $\mathrm{PCl}$, ACEl or ARB, beta-blockade, statin, Paris thrombotic risk score, and Paris bleeding risk score 


\section{a All-cause death and new Q-wave MI}

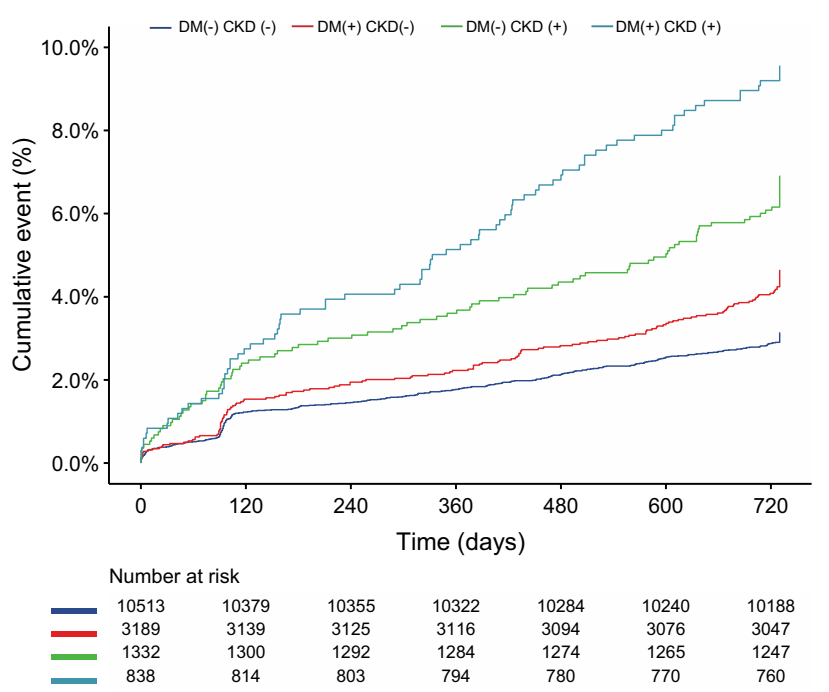

b BARC type 3 or 5 bleeding events

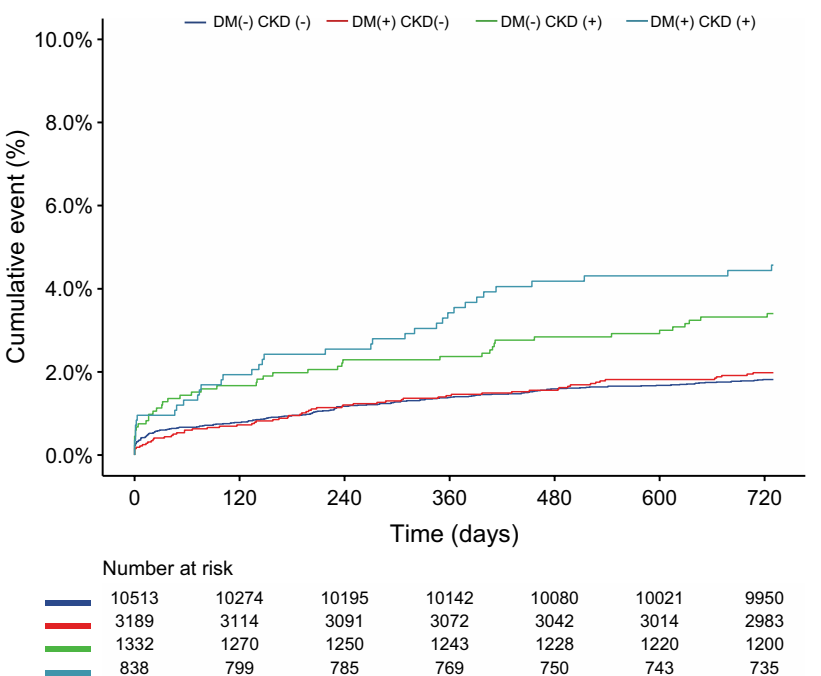

Fig. 2 Clinical events shown by Kaplan-Meier curves. a All-cause mortality and new Q-wave Ml; b Bleeding Academic Research Consortium (BARC)-defined type 3 or 5 bleeding events;

subgroups (Fig. 3a, b, and Table 3). Although not statistically significant, the absolute risk reduction of the primary endpoint gradually increased in the following order of DM-/CKD-, DM-/CKD+, DM+/CKD-, DM+/ $\mathrm{CKD}+(0.3 \%, 1.0 \%, 1.1 \%$, and $2.3 \%)$ in patients receiving the experimental regimen.

Among the $\mathrm{DM}+/ \mathrm{CKD}+$ patients, the experimental regimen was associated with lower rates of POCE $(20.6 \%$ versus $25.9 \%$, $H R \quad 0.74 ; 95 \% \mathrm{CI}[0.55-0.99], \mathrm{p}=0.043$, $\left.\mathrm{p}_{\text {interaction }}=0.155\right)$ and NACE $(22.7 \%$ versus $28.3 \%$, HR $0.75 ; 95 \% \mathrm{CI}[0.56-0.99], \mathrm{p}=0.044, \mathrm{p}_{\text {interaction }}=0.310$ ), which were mainly driven by lower rates of any revascularization (11.5\% versus 15.6\%; adjusted HR 0.67; 95\%CI [0.45-0.99], $\left.\mathrm{P}=0.042, \mathrm{p}_{\text {interaction }}=0.286\right)$ and TVR $(6.1 \%$ versus 10.0\%; adjusted $\mathrm{HR} 0.56$; 95\% CI [0.33-0.93], $\mathrm{P}=0.026, \mathrm{p}_{\text {interaction }}=0.238$; Fig. $3 \mathrm{c}-\mathrm{f}$, and Table 3 ), as compared with the reference regimen. The numbers needed-to-treat to reduce a POCE, NACE, any revascularization and TVR event were 19, 18, 24 and 25, respectively. Additional sensitivity analyses were performed to investigated the treatment effects of experimental regimen in patients who were adherent to the allocated medications, in ACS patients, and in Stable CAD patients, respectively. The results are shown in Additional file 1: Table S5-S7.

\section{Landmark analysis}

Given that according to the study protocol, the reference treatment strategy arm received conventional 12-month DAPT (Clopidogrel/Ticagrelor was stopped at 12-month) followed by 12- month aspirin monotherapy, we performed a landmark analysis at 365 days after the index procedure to specifically analyze the impact of P2Y12 discontinuation in the reference strategy. The results showed that among $\mathrm{DM}+/ \mathrm{CKD}+$ patients, between 0-365 days after randomization, the experimental and reference regimen had similar rates of all investigated endpoints (Additional file 1: Table S4 and Fig. S2 ), whereas between 365-730 days after randomization, compared with the reference regimen, the experimental regimen was associated with significantly lower rates of POCE (5.8\% versus 11.0\%, HR 0.49; 95\% CI $\left.[0.29-0.82], \mathrm{p}=0.007, \mathrm{p}_{\text {interaction }}=0.040\right)$, NACE $(5.8 \%$ versus $11.2 \%$, HR 0.48 ; 95\% CI [0.29-0.82], $\mathrm{p}=0.007$, $\left.\mathrm{p}_{\text {interaction }}=0.013\right)$, any revascularization $(2.3 \%$ versus 6.6\%, adjusted HR 0.29; 95\% CI [0.13-0.65], $\mathrm{P}=0.003$, $\left.\mathrm{p}_{\text {interaction }}=0.056\right)$ and TVR $(1.4 \%$ versus $2.9 \%$, adjusted HR 0.29; 95\% CI [0.09-0.91], P =0.033, $\mathrm{p}_{\text {interaction }}=0.112$ ) (Table 4 and Additional file 1: Fig. S2). The rate of BARC type 3 or 5 bleeding events $(0.7 \%$ versus $1.5 \%, \mathrm{P}=0.331)$ was similar between the two antiplatelet regimens between 365 and 730 days after randomization.

\section{Discussion}

The main findings of this post hoc analysis of the GLOBAL LEADERS trial can be summarized as follows:

1) The concomitant presence of DM and CKD is not uncommon in an "all-comers" trial, representing 21\% of the patients with DM, and 5\% of the overall study population. 


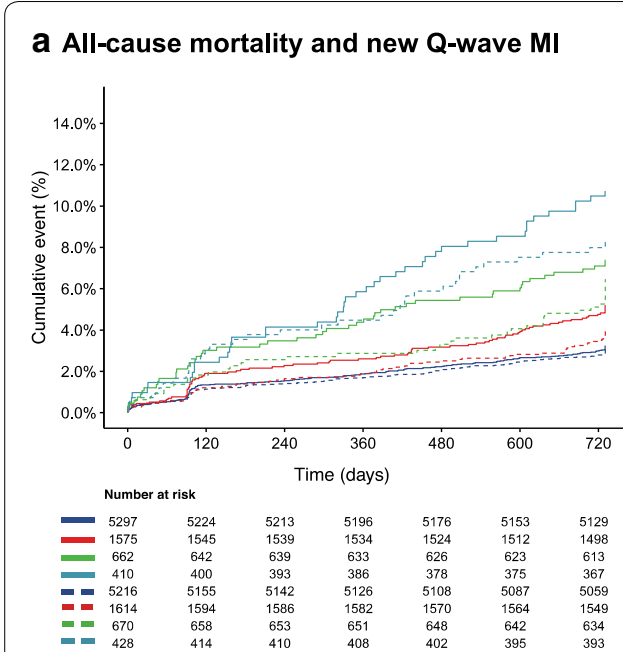

b BARC 3 or 5

\section{Any Revascularization}
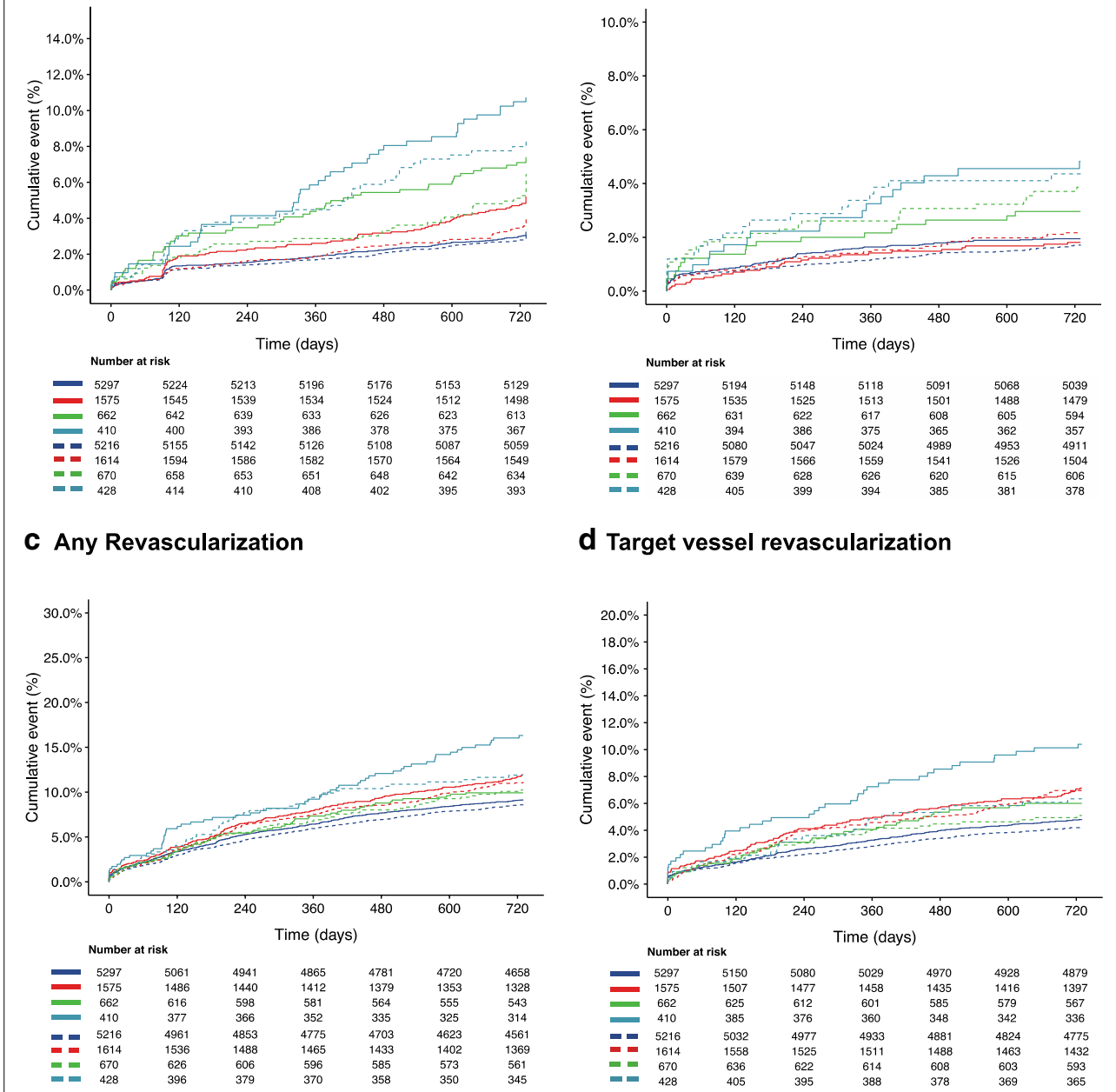

\section{d Target vessel revascularization}

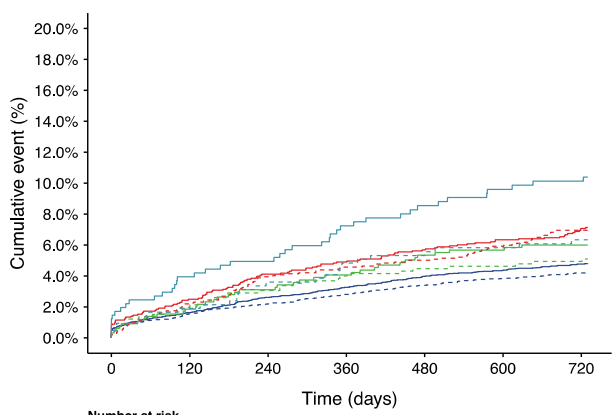

Table Legends

Reference Treatment

- DM(-) CKD (-) - DM(+) CKD (-)

$-\mathrm{DM}(-) \mathrm{CKD}(+)-\mathrm{DM}(+) \mathrm{CKD}(+)$

\section{Experimental Treatment}

- - DM (-) CKD (-) -- DM(+) CKD (-)

$--\operatorname{DM}(-) \mathrm{CKD}(+)--\mathrm{DM}(+) \mathrm{CKD}(+)$

\section{e POCE}
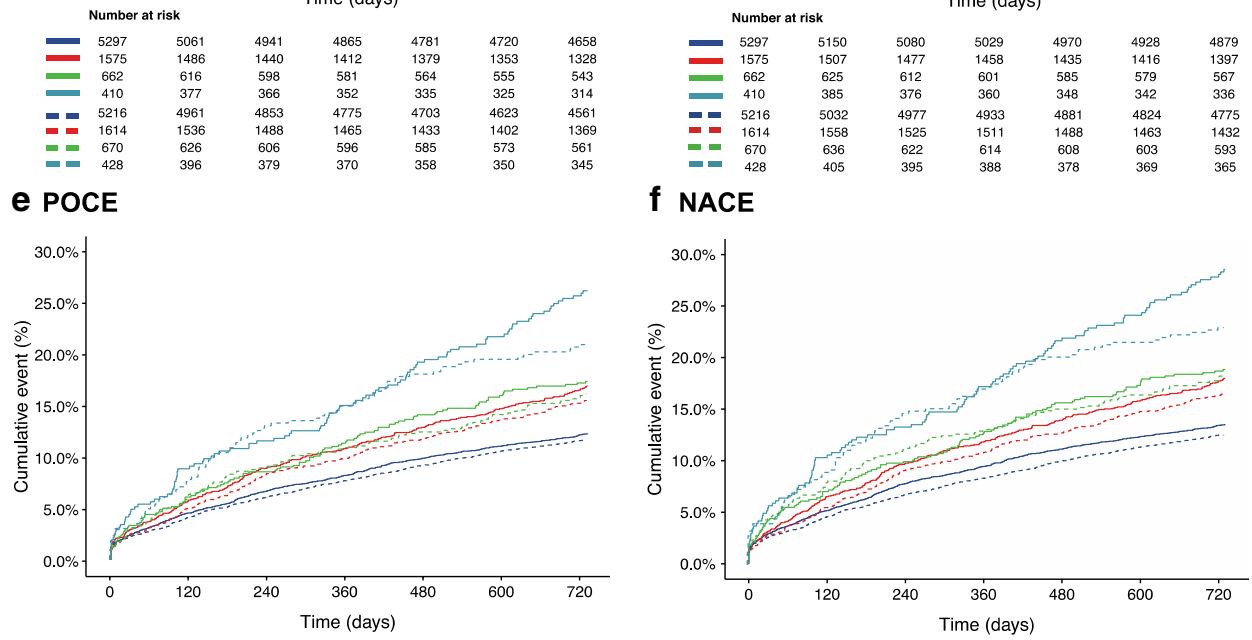

f NACE
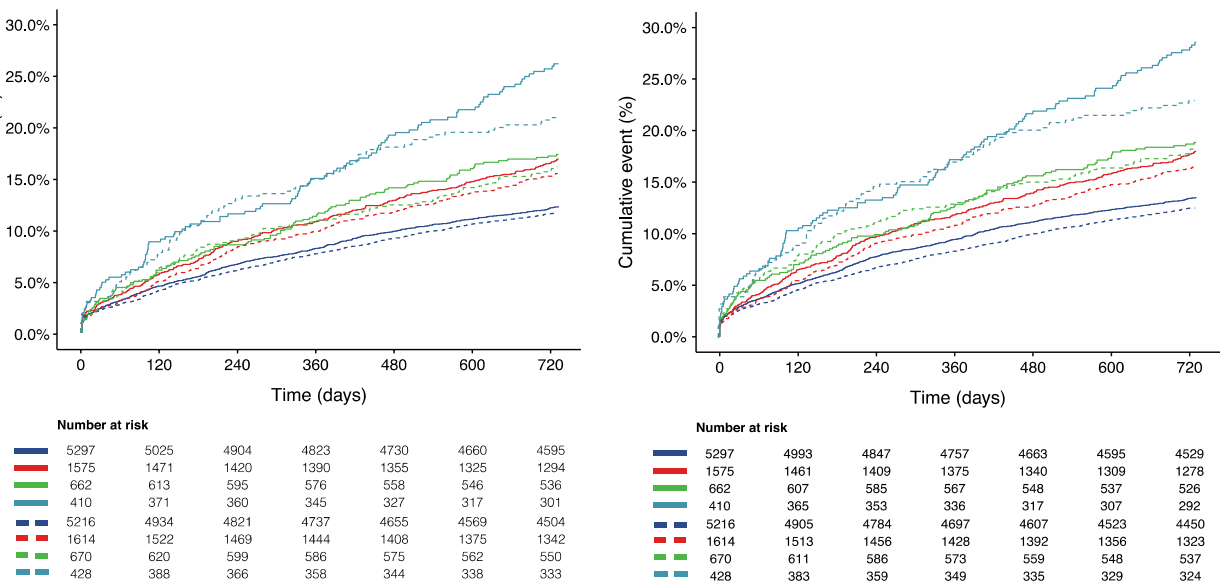

Fig. 3 Kaplan-Meier curves showing the clinical events according to treatment regimen and DM/CKD status. a All-cause mortality and new Q-wave Ml; b Bleeding Academic Research Consortium (BARC)-defined type 3 or 5 bleeding events; c Any revascularization; $\mathbf{d}$ Target vessel revascularization; e POCE; f NACE; 
Table 3 Forest plot of the endpoints according to treatment regimen and DM/CKD status

\begin{tabular}{|c|c|c|c|c|c|c|}
\hline & DM/CKD group & $\begin{array}{c}\text { Experimental } \\
\text { Treatment }\end{array}$ & $\begin{array}{l}\text { Reference } \\
\text { Treatment }\end{array}$ & $\begin{array}{c}\text { Hazard ratio } \\
(95 \% \mathrm{Cl})\end{array}$ & $P$ value & Pinteraction \\
\hline $\begin{array}{l}\text { All-cause mortality } \\
\text { or New Q-wave MI }\end{array}$ & $\begin{array}{c}\mathrm{DM}(-) \text { CKD }(-) \\
\operatorname{DM}(+) \operatorname{CKD}(-) \\
\operatorname{DM}(-) \text { CKD }(+) \\
\operatorname{DM}(+) \text { CKD }\left(^{+}\right)\end{array}$ & $\begin{array}{l}158 / 5216(3 \%) \\
66 / 1614(4.1 \%) \\
43 / 670(6.4 \%) \\
36 / 428(8.4 \%)\end{array}$ & $\begin{array}{l}172 / 5297(3.2 \%) \\
82 / 1575(5.2 \%) \\
49 / 662(7.4 \%) \\
44 / 410(10.7 \%)\end{array}$ & $\begin{array}{l}0.95(0.76-1.18) \\
0.78(0.56-1.1) \\
0.85(0.56-1.28) \\
0.75(0.47-|.| 8)\end{array}$ & $\begin{array}{l}0.539 \\
0.105 \\
0.813 \\
0.087\end{array}$ & 0.228 \\
\hline All-cause mortality & $\begin{array}{c}\mathrm{DM}(-) \text { CKD }(-) \\
\operatorname{DM}(+) \text { CKD }(-) \\
\operatorname{DM}(-) \text { CKD }(+) \\
\operatorname{DM}(+) \text { CKD }(+)\end{array}$ & $\begin{array}{l}112 / 5216(2.1 \%) \\
49 / 1614(3 \%) \\
32 / 670(4.8 \%) \\
30 / 428(7 \%)\end{array}$ & $\begin{array}{l}114 / 5297(2.2 \%) \\
58 / 1575(3.7 \%) \\
42 / 662(6.3 \%) \\
37 / 410(9 \%)\end{array}$ & $\begin{array}{l}1.05(0.81-1.38) \\
0.84(0.57-1.24) \\
0.74(0.46-1.19) \\
0.72(0.44-1.19)\end{array}$ & $\begin{array}{l}0.919 \\
0.222 \\
0.49 \\
0.118\end{array}$ & 0.051 \\
\hline Myocardial infarction & $\begin{array}{c}\mathrm{DM}(-) \text { CKD }(-) \\
\operatorname{DM}(+) \operatorname{CKD}(-) \\
\operatorname{DM}(-) \operatorname{CKD}(+) \\
\operatorname{DM}(+) \operatorname{CKD}(+)\end{array}$ & $\begin{array}{l}130 / 5216(2.5 \%) \\
64 / 1614(4 \%) \\
28 / 670(4.2 \%) \\
26 / 428(6.1 \%)\end{array}$ & $\begin{array}{l}143 / 5297(2.7 \%) \\
55 / 1575(3.5 \%) \\
24 / 662(3.6 \%) \\
27 / 410(6.6 \%)\end{array}$ & $\begin{array}{l}0.93(0.73-1.19) \\
1.19(0.82-1.73) \\
1.11(0.62-1.96) \\
1.05(0.6-1.84)\end{array}$ & $\begin{array}{l}0.543 \\
0.36 \\
0.613 \\
0.862\end{array}$ & 0.487 \\
\hline Any Revascularization & $\begin{array}{r}\mathrm{DM}(-) \text { CKD (-) } \\
\operatorname{DM}(+) \text { CKD }(-) \\
\operatorname{DM}(-) \text { CKD (+) } \\
\operatorname{DM}(+) \text { CKD }(+)\end{array}$ & $\begin{array}{l}439 / 5216(8.4 \%) \\
176 / 1614(10.9 \%) \\
66 / 670(9.9 \%) \\
49 / 428(11.4 \%)\end{array}$ & $\begin{array}{l}478 / 5297(9 \%) \\
184 / 1575(11.7 \%) \\
63 / 662(9.5 \%) \\
64 / 410(15.6 \%)\end{array}$ & $\begin{array}{l}0.97(0.85-1.11) \\
0.96(0.77-1.19) \\
1.04(0.73-1.49) \\
0.67(0.45-0.99)\end{array}$ & $\begin{array}{l}0.718 \\
0.742 \\
0.819 \\
0.038\end{array}$ & 0.228 \\
\hline $\begin{array}{l}\text { Target vessel } \\
\text { Revascularization }\end{array}$ & $\begin{array}{r}\mathrm{DM}(-) \text { CKD (-) } \\
\mathrm{DM}(+) \text { CKD (-) } \\
\mathrm{DM}(-) \text { CKD (+) } \\
\mathrm{DM}(+) \text { CKD }\left(^{+}\right)\end{array}$ & $\begin{array}{l}215 / 5216(4.1 \%) \\
110 / 1614(6.8 \%) \\
33 / 670(4.9 \%) \\
26 / 428(6.1 \%)\end{array}$ & $\begin{array}{l}251 / 5297(4.7 \%) \\
110 / 1575(7 \%) \\
38 / 662(5.7 \%) \\
41 / 410(10 \%)\end{array}$ & $\begin{array}{l}0.9(0.75-1.09) \\
1.03(0.78-1.35) \\
0.86(0.53-1.39) \\
0.56(0.33-0.93)\end{array}$ & $\begin{array}{l}0.29 \\
0.787 \\
0.554 \\
0.026\end{array}$ & 0.235 \\
\hline BARC type 3 or 5 bleeding & $\begin{array}{c}\mathrm{DM}(-) \text { CKD }(-) \\
\operatorname{DM}(+) \text { CKD }(-) \\
\operatorname{DM}(-) \text { CKD (+) } \\
\operatorname{DM}(+) \text { CKD }\left(^{+}\right)\end{array}$ & $\begin{array}{l}86 / 5216(1.6 \%) \\
34 / 1614(2.1 \%) \\
25 / 670(3.7 \%) \\
18 / 428(4.2 \%)\end{array}$ & $\begin{array}{l}102 / 5297(1.9 \%) \\
28 / 1575(1.8 \%) \\
19 / 662(2.9 \%) \\
19 / 410(4.6 \%)\end{array}$ & $\begin{array}{l}0.91(0.68-1.22) \\
1.15(0.69-1.91) \\
1.45(0.79-2.68) \\
0.86(0.45-1.64)\end{array}$ & $\begin{array}{l}0.506 \\
0.626 \\
0.215 \\
0.587\end{array}$ & 0.643 \\
\hline BARC type 2 bleeding & $\begin{array}{r}\operatorname{DM}(-) \operatorname{CKD}(-) \\
\operatorname{DM}(+) \operatorname{CKD}(-) \\
\operatorname{DM}(-) \operatorname{CKD}(+) \\
\operatorname{DM}(+) \operatorname{CKD}(+)\end{array}$ & $\begin{array}{l}236 / 5216(4.5 \%) \\
83 / 1614(5.1 \%) \\
42 / 670(6.3 \%) \\
31 / 428(7.2 \%)\end{array}$ & $\begin{array}{l}253 / 5297(4.8 \%) \\
72 / 1575(4.6 \%) \\
40 / 662(6 \%) \\
23 / 410(5.6 \%)\end{array}$ & $\begin{array}{l}0.95(0.8-1.14) \\
1.18(0.85-1.62) \\
1.08(0.69-1.7) \\
1.21(0.69-2.12)\end{array}$ & $\begin{array}{l}0.602 \\
0.327 \\
0.832 \\
0.494\end{array}$ & 0.248 \\
\hline BARC type 2,3 or 5 bleeding & $\begin{array}{l}\text { D } \operatorname{DM}(-) \operatorname{CKD}(-) \\
\operatorname{DM}(+) \operatorname{CKD}(-) \\
\operatorname{DM}(-) \operatorname{CKD}(+) \\
\operatorname{DM}(+) \operatorname{CKD}(+)\end{array}$ & $\begin{array}{l}310 / 5216(5.9 \%) \\
112 / 1614(6.9 \%) \\
62 / 670(9.3 \%) \\
44 / 428(10.3 \%)\end{array}$ & $\begin{array}{l}337 / 5297(6.4 \%) \\
94 / 1575(6 \%) \\
56 / 662(8.5 \%) \\
40 / 410(9.8 \%)\end{array}$ & $\begin{array}{l}0.95(0.82-1.12) \\
1.19(0.9-1.58) \\
1.16(0.8-1.68) \\
0.98(0.63-1.51)\end{array}$ & $\begin{array}{l}0.556 \\
0.212 \\
0.45 \\
0.913\end{array}$ & 0.395 \\
\hline $\begin{array}{l}\text { POCE - all-cause death, } \\
\text { any stroke, any myoca- } \\
\text { rdial infarction or any } \\
\text { revascularization }\end{array}$ & $\begin{array}{c}\mathrm{DM}(-) \operatorname{CKD}(-) \\
\operatorname{DM}(+) \operatorname{CKD}(-) \\
\operatorname{DM}(-) \operatorname{CKD}(+) \\
\operatorname{DM}(+) \operatorname{CKD}(+)\end{array}$ & $\begin{array}{l}598 / 5216(11.5 \%) \\
248 / 1614(15.4 \%) \\
106 / 670(15.8 \%) \\
88 / 428(20.6 \%)\end{array}$ & $\begin{array}{l}644 / 5297(12.2 \%) \\
263 / 1575(16.7 \%) \\
113 / 662(17.1 \%) \\
106 / 410(25.9 \%)\end{array}$ & $\begin{array}{l}0.98(0.88-|.|) \\
0.94(0.78-|.| 2) \\
0.94(0.7|-| .23) \\
0.74(0.55-0.99)\end{array}$ & $\begin{array}{l}0.806 \\
0.496 \\
0.916 \\
0.033\end{array}$ & 0.125 \\
\hline $\begin{array}{l}\text { NACE- POCE and } \\
\text { BARC } 3 \text { or } 5 \text { bleeding }\end{array}$ & $\begin{array}{c}\mathrm{DM}(-) \operatorname{CKD}(-) \\
\mathrm{DM}(+) \operatorname{CKD}(-) \\
\operatorname{DM}(-) \operatorname{CKD}(+) \\
\operatorname{DM}(+) \operatorname{CKD}(+)\end{array}$ & $\begin{array}{l}649 / 5216(12.4 \%) \\
267 / 1614(16.5 \%) \\
122 / 670(18.2 \%) \\
97 / 428(22.7 \%)\end{array}$ & $\begin{array}{l}711 / 5297(13.4 \%) \\
281 / 1575(17.8 \%) \\
123 / 662(18.6 \%) \\
116 / 410(28.3 \%)\end{array}$ & $\begin{array}{l}0.96(0.86-1.07) \\
0.94(0.79-1.12) \\
1.02(0.79-1.32) \\
0.75(0.56-0.99)\end{array}$ & $\begin{array}{l}0.544 \\
0.508 \\
0.599 \\
0.034\end{array}$ & 0.261 \\
\hline \multicolumn{5}{|r|}{ Favor Experimental } & \multicolumn{2}{|c|}{ Favor Reference } \\
\hline
\end{tabular}

Adjusted to age, sex, body mass index (BMI), clinical presentation (ACS versus stable CAD), stroke, peripheral vascular disease (PVD), chronic obstructive pulmonary disease (COPD), previous PCI, hypercholesterolemia, hypertension, current smoking status, complex PCI, ACEI or ARB, beta-blockade, statin, Paris thrombotic risk score, and Paris bleeding risk score

2) Up to two years post-PCI, there was a gradient in the thrombotic and bleeding risk among patients stratified according to the presence or absence of DM or CKD, with the highest risk found among subjects having both comorbidities.

3) In patients with both DM and CKD, the primary endpoint (all-cause mortality or new Q-wave MI) or the key safety secondary endpoint (BARC type 3 or 5 bleeding) did not differ significantly between the experimental and the reference regimens. Notwithstanding, the experimental regimen was associated with lower rates of POCE and NACE, mainly driven by repeat revascularization. 
Table 4 Forest plot of the endpoints by landmark analyses (365-730 days)

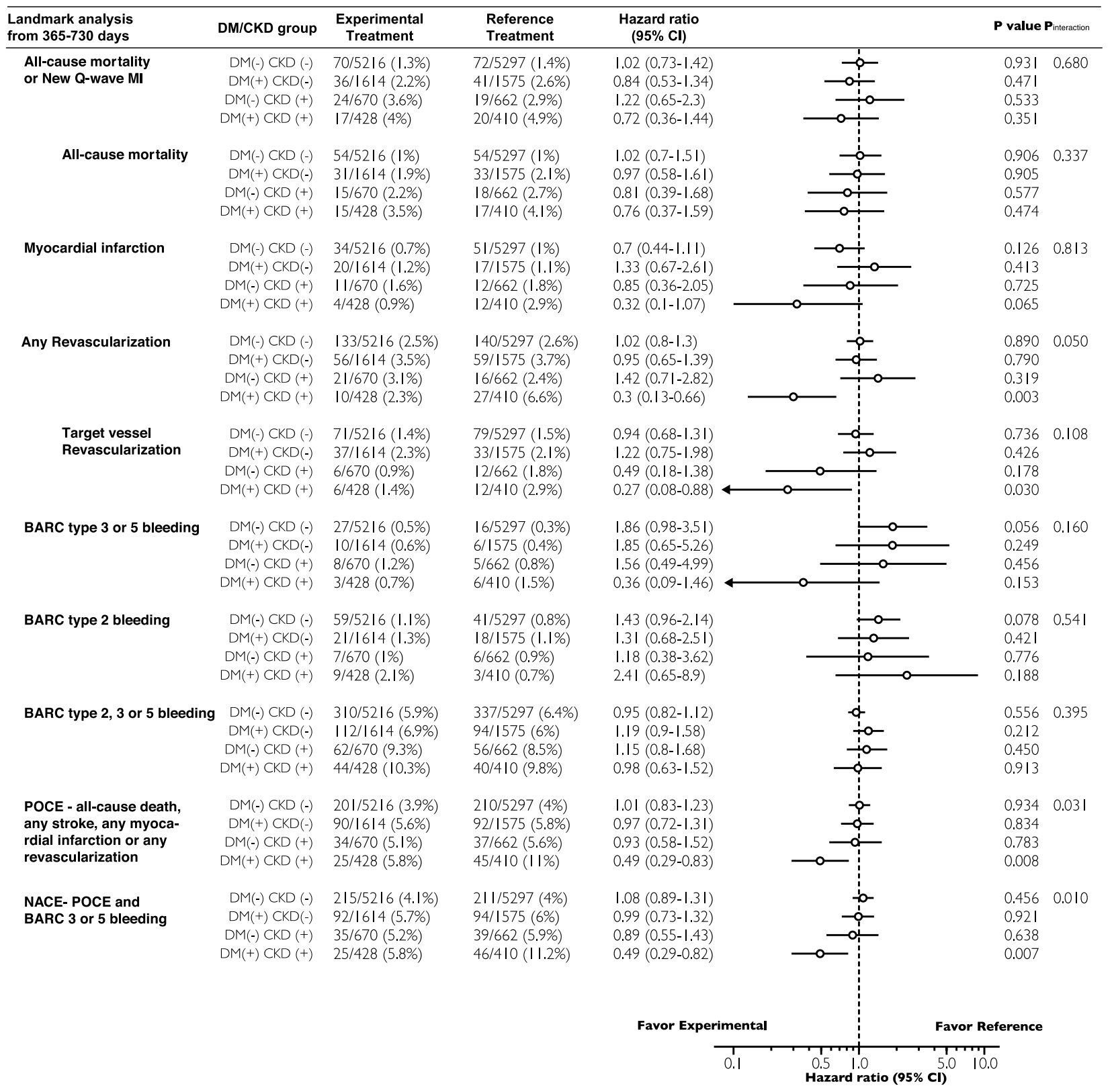

\section{The prevalence and prognosis of CAD patients with DM} and CKD

Both DM and CKD are independently associated with an increased risk of cardiovascular ischemic events, which can be attributed to patients' pro-thrombotic and pro-inflammatory status [2,3]. These two risk factors of coronary heart disease have also been shown to synergistically amplify the hazards when they co-exist. Reports published nearly two decades ago showed that mortality rates one year after successful PCI in DM patients with moderate and severe CKD were respectively, 5- and 12-times higher when compared to patients with normal renal function [21]. A subgroup analysis of the PLATO trial -a trial conducted over a decade ago [22], showed that patients with the combination of DM and CKD had a greater than threefold increase in the risk of mortality [6]. In the contemporary GLOBAL LEADERS trial, we found that despite the progressive improvements in stent design and secondary preventive pharmacotherapies, patients with both DM and CKD still had a 2.1-fold higher risk of mortality, 1.6-fold higher risk of repeat revascularization, and 1.6-fold higher risk of BARC 3 or 
5 bleeding, compared with patients without these risk factors. Although these results suggest that the hazards of having both comorbidities have somewhat attenuated over the years, patients with both DM and CKD were still at high risk of ischemic and bleeding events. These observations underscore the need to identify novel therapeutic approaches that can reduce the risks in this specific population.

In the current analysis, we found that $20.8 \%$ (838/4027) of DM patients had CKD. This proportion is relatively similar among some pivotal cardiovascular RCTs. For instance, in the PLATO trial that investigated adjunctive antiplatelet pharmacotherapy in patients with acute coronary syndromes [6], the percentage of DM patients who had CKD was $22.0 \%(1058 / 3807)$. In the SYNTAX trial, which tested the optimal revascularization technique in patients with complex coronary lesions, the proportion was $20.8 \%$ (85/408, unpublished data). However, data from a German national database [23] and two dedicated registries (Diabetes-Patienten-Verlaufsdokumentation and DIabetes Versorgungs-Evaluation) [24] suggested that approximately $40-50 \%$ of individuals with DM have comorbid CKD. Therefore, when compared with these population-based studies, the $\mathrm{DM}+/ \mathrm{CKD}+$ population in the GLOBAL LEADERS and some cardiovascular RCTs might be underrepresented, or conversely these registries with specific inclusion criteria may have an overrepresentation of the syndrome.

DAPT strategy for $D M+/ C K D+$ patients $(0-1$ year post $P C l)$ The optimal DAPT strategy for DM+/CKD + patients remains a matter of debate owing to scarce evidence. Generally, $\mathrm{DM}+/ \mathrm{CKD}+$ patients are at high bleeding risk [25]. In the GLOBAL LEADERS population, 71.0\% of the $\mathrm{DM}+/ \mathrm{CKD}+$ patients had a PRECISE-DAPT score of 25 or more. As suggested by the 2018 European Society of Cardiology guidelines on Myocardial Revascularization [1], patients with high bleeding risk (PRECISEDAPT score of 25 as the cutoff point) should discontinue DAPT after 3- (in stable CAD) or 6-months (in ACS) post-PCI to reduce the risk of bleeding; however, $\mathrm{DM}+1$ CKD + patients were also at high thrombotic risk (73.4\% of these patients had a Paris thrombotic risk score of $>5$ ). Indeed, a short DAPT strategy would reduce bleeding events, but at the same time, might plausibly augment the thrombotic risk [26, 27].

Considering the dilemma of DAPT duration, the strategy of ticagrelor monotherapy has been proposed as a means to reduce the risk of bleeding while maintaining a similar risk of thrombotic events after PCI. The TWILIGHT trial $[8,28]$, in which either DM or CKD constituted an enrichment criteria according to the protocol (2620 pts with DM and 1145 pts with CKD in the
TWILIGHT trial), has compared 3-month DAPT followed by 12-month ticagrelor monotherapy after PCI with standard DAPT strategy. The results showed a significant reduction of BARC type 2, 3 or 5 bleeding events in the ticagrelor monotherapy arm, while demonstrating a similar risk of the composite secondary endpoint of all-cause death, non-fatal MI, or stroke. Compared with the TWILIGHT trial, the current study showed that in $\mathrm{DM}+/ \mathrm{CKD}+$ patients, ticagrelor monotherapy strategy in the first year had similar rates of all-cause mortality, MI or revascularization, as well as the rate of BARC type 2, 3 or 5 bleeding events, compared with the standard DAPT strategy. These results showed that although the thrombotic risks were higher in DM+/CKD + patients, ticagrelor monotherapy (or the "aspirin-free strategy") might not be associated with increased thrombotic events compared with the standard DAPT.

\section{Prolonged ticagrelor monotherapy for secondary prevention ( 1 year post $\mathrm{PCl}$ )}

To date, there is no evidence elaborating the optimal antiplatelet medication for the secondary prevention of the DM+/CKD+patients post PCI. Alike other patients, those patients are now generally treated with aspirin lifelong for secondary prevention. Whether ticagrelor represents a worse, alternative, or better choice still debatable. In DM patients with stable CAD and a history of PCI), results of the THEMIS-PCI trial [29, 30] have demonstrated that compared to aspirin for secondary prevention, ticagrelor reduced the ischemic endpoint of cardiovascular death, MI, and stroke with modestly increasing the bleeding events. In total, ticagrelor improved the net clinical benefit $(9.3 \%$ versus $11.0 \%$, $\mathrm{HR}=0.85,95 \% \mathrm{CI} 0.75-0.95, \mathrm{p}=0.005)$ in the THEMISPCI population. For the DM+/CKD + patients, whether it is legitimate to simply apply to the recommendation for DM patients (such as the results of the TWILIGHT and the THEMIS-PCI trial), is somehow based on empirical experiences. So far, there is no specific narrative in the consensus or guideline helping the clinician to make the decision. The current analysis found that in $\mathrm{DM}+/$ CKD + patients, compared with aspirin monotherapy, the ticagrelor monotherapy had similar BARC type 2, 3 or 5 bleeding events, meanwhile, was associated with lower rates of POCE and NACE, which were predominantly confined to reductions in any revascularization or TVR events that occurred during the second year of the trial.

Legitimately, like the THEMIS-PCI trial, prolonging the use of ticagrelor would increase the risk of bleeding. The neutral statistical findings in our analysis regarding BARC type 2, 3 or 5 bleeding events might be due to play of chance or the relatively low sample size (although it is 
one of the largest trials investigating such issue). However, the improved net clinical benefit of the ticagrelor monotherapy shown in our analysis and the THEMISPCI trial supported that prolonged ticagrelor might be a reasonable treatment option for $\mathrm{DM}$ or $\mathrm{DM}+1$ $\mathrm{CKD}+$ patients regarding secondary prevention. Of note, given the inherent limitations of sub-analyses, our findings cannot make strong inferences nor necessitate changes in clinical practice.

\section{Limitations}

The following limitations have to be considered in the present analysis. (1) Given that the two antiplatelet strategies did not differ significantly with regard to rates of the primary endpoint in the overall trial [7], and the post hoc nature of the study, all reported analyses have to be considered strictly exploratory. (2) The randomization in the GLOBAL LEADERS trial was not stratified according to the presence of DM or CKD, therefore some imbalances between the randomized groups may exist among the four sub-categories. Although multivariable adjusted Cox proportional hazard models were performed to try to estimate the true treatment effects of the different regimens, the usual deficiency for observational studies exists, such as the inability to include all relevant confounders especially those unmeasured, causing bias which cannot be adjusted.

\section{Conclusions}

The present analysis showed that in a contemporary PCI cohort, patients with DM and CKD are at markedly increased risk of long-term thrombotic and bleeding events, compared with patients one or neither of these risk factors. In patients with both comorbidities, ticagrelor monotherapy was not associated with a lower rate of the primary endpoint (all-cause mortality or new Q-wave MI) or bleeding (BARC type 3 or 5 bleeding), but was associated with a lower rate of POCE and NACE, which was mainly driven by the lower rate of any revascularization.

\section{Supplementary information}

Supplementary information accompanies this paper at https://doi. org/10.1186/s12933-020-01153-x.

Additional file 1: Table S1. Forest plot of the ischemic endpoints according to treatment regimen and DM/CKD status. Table S2. Forest plot of the bleeding endpoints according to treatment regimen and DM/CKD status. Table S3. Forest plot of sensitivity analyses (stage II to V CKD by KDIGO classification) showing outcomes according to treatment regimen and DM/CKD status. Table S4. Forest plot of landmark analyses (0-365 days) showing outcomes of reference versus experimental treatment according to DM/CKD status. Table S5. Forest plot of sensitivity analyses (subjects who were adherent to the allocated medication) showing outcomes according to treatment regimen and DM/CKD status. Table S6. Forest plot of sensitivity analyses (ACS patients) showing outcomes according to treatment regimen and DM/CKD status. Table S7. Forest plot of sensitivity analyses (Stable CAD patients) showing outcomes according to treatment regimen and DM/CKD status. Table S8. Forest plot of sensitivity analyses (Propensity score adjusted Cox regression model) showing outcomes according to treatment regimen and DM/CKD status. Figure S1. Distribution of propensity score. Figure S2. Kaplan-Meier curves of the landmark analysis showing outcomes of treatment regimen according to DM/CKD status.

\section{Abbreviations}

ACS: Acute coronary syndrome; ARC: Academic Research Consortium; BARC : Bleeding Academic Research Consortium; BMI: Body mass index; CABG: Coronary artery bypass graft surgery; CAD: Coronary artery disease; CKD: Chronic Kidney Disease; COPD: Chronic Obstructive Pulmonary Disease; DAPT: Dual-antiplatelet therapy; DES: Drug Eluting Stent; DM: Diabetes Mellitus; ECG: Electrocardiogram/electrocardiography; eGFR: Estimated glomerular filtration rate; MDRD: Modification of Diet in Renal Disease; NACE: Net adverse clinical events; NSTEMI: Non-ST-segment elevation MI; PCI: Percutaneous Coronary Intervention; POCE: Patient-oriented Composite Endpoint; ST: Stent thrombosis; STEMI: ST elevation MI; TIMI: Thrombolysis In Myocardial Infarction; TLF: Target Lesion Failure; TV MI: Target vessel Myocardial Infarction; TVR: Target vessel revascularization.

\section{Acknowledgements}

Not applicable.

\section{Authors' contributions}

CG, MT, KT, HK, RT, HH, MO, and DA analyzed and interpreted data, wrote the first draft of the article and contributed to all revisions. GM, SG, MH, TS, PV, RG gathered and cleaned the data. MV, SW, CH, PS, YO, PWS gathered and interpreted data and contributed to critical revision of the manuscript. All authors read and approved the final manuscript.

\section{Funding}

This work was supported by the European Clinical Research Institute, which received unrestricted grants from Biosensors International, AstraZeneca, and the Medicines Company.

\section{Availability of data and materials}

The datasets generated and/or analysed during the current study are not publicly available but are available from the corresponding author on reasonable request.

\section{Ethics approval and consent to participate}

The trial was approved by the institutional review board at each center and followed the ethical principles of the Declaration of Helsinki. All patients provided written informed consent prior to participation in the trial.

\section{Consent for publication}

All authors have participated in the work and have reviewed and agree with the content of the article. None of the article contents are under consideration for publication in any other journal or have been published in any journal. No portion of the text has been copied from other material in the literature (unless in quotation marks, with citation). We are aware that it is the authors responsibility to obtain permission for any figures or tables reproduced from any prior publications, and to cover fully any costs involved. Such permission must be obtained prior to final acceptance.

\section{Competing interests}

Dr. Steg received grants and personal fees from Bayer/Janssen, grants and personal fees from Merck, grants and personal fees from Sanofi, grants and personal fees from Amarin, personal fees from Amgen, personal fees from Bristol Myers Squibb, personal fees from Boehringer-Ingelheim, personal fees from Pfizer, personal fees from Novartis, personal fees from Regeneron, personal fees from Lilly, personal fees from AstraZeneca, grants, personal fees and non-financial support from Servier, outside the submitted work. Dr. Hamm received advisory Board fees from AstraZeneca. Dr. van Geuns received speakers fee from Abbott Vascular and Boston Scientific. Dr. Onuma reports being a 
member of advisory board of Abbott vascular. Dr. Serruys reports personal fees from Biosensors, personal fees from Cardialysis, personal fees from Medtronic, personal fees from Micel Technologies, personal fees from Sinomedical Sciences Technology, personal fees from Philips/Nolcano, personal fees from Xeltis, personal fees from HeartFlow, outside the submitted work. Dr. Angiolillo has received payment as an individual for: reports receiving payments as an individual for: a) Consulting fee or honorarium from Amgen, Aralez, AstraZeneca, Bayer, Biosensors, Boehringer Ingelheim, Bristol-Myers Squibb, Chiesi, Daiichi-Sankyo, Eli Lilly, Haemonetics, Janssen, Merck, PhaseBio, PLx Pharma, Pfizer, Sanofi, and The Medicines Company; b) Participation in review activities from CeloNova and St. Jude Medical. Institutional payments for grants from Amgen, AstraZeneca, Bayer, Biosensors, CeloNova, CSL Behring, DaiichiSankyo, Eisai, Eli-Lilly, Gilead, Idorsia, Janssen, Matsutani Chemical Industry Co., Merck, Novartis, Osprey Medical, and Renal Guard Solutions.

\section{Author details}

${ }^{1}$ Department of Cardiology, Xijing hospital, Xi'an, China. ${ }^{2}$ Department of Cardiology, Radboud University, Nijmegen, The Netherlands. ${ }^{3}$ First Department of Cardiology, Medical University of Warsaw, Warsaw, Poland. ${ }^{4}$ Erasmus Medical Center, Erasmus University, Rotterdam, The Netherlands. ${ }^{5}$ Amsterdam UMC, University of Amsterdam, Amsterdam, The Netherlands. ${ }^{6}$ Sorbonne University, ACTION Study Group, Institute of Cardiology, Pitié-Salpêtrière Hospital, Paris, France. ${ }^{7}$ Department of Cardiology, Royal Blackburn Hospital, Blackburn, UK. ${ }^{8}$ Department of Cardiology, Rheinland Klinikum Neuss, Lukaskrankenhaus, Neuss, Germany. ${ }^{9}$ OLVG, Amsterdam, Netherlands. ${ }^{10}$ Department of Cardiology and Critical Care Medicine, Hartcentrum Hasselt, Jessa Ziekenhuis, Hasselt, Belgium. ${ }^{11}$ Department of Cardiology, Bern University Hospital, Bern, Switzerland. ${ }^{12}$ Kerckhoff Heart Center, Bad Nauheim, Germany. ${ }^{13}$ FACT, French Alliance for Cardiovascular Trials, Paris, France. ${ }^{14}$ Hôpital Bichat, AP-HP, Paris, France. ${ }^{15}$ Department of Cardiology, National University of Ireland Galway, Galway, Ireland. ${ }^{16}$ Division of Cardiology, University of Florida College of Medicine, Jacksonville, FL, USA. ${ }^{17} \mathrm{NHLI}$, Imperial College London, London, UK. ${ }^{18}$ Interventional Medicine and Innovation, National University of Ireland Galway, P.O. University Road, Galway H91 TK33, Ireland.

Received: 5 July 2020 Accepted: 5 October 2020

Published online: 16 October 2020

\section{References}

1. Neumann FJ, Sousa-Uva M, Ahlsson A, Alfonso F, Banning AP, Benedetto U, Byrne RA, Collet JP, FalkV, Head SJ, et al. 2018 ESC/EACTS Guidelines on myocardial revascularization. Eur Heart J. 2019;40(2):87-165.

2. Capodanno D, Angiolillo DJ. Antithrombotic therapy in patients with chronic kidney disease. Circulation. 2012;125(21):2649-61.

3. Ferreiro $\mathrm{J}$, Angiolillo DJ. Diabetes and antiplatelet therapy in acute coronary syndrome. Circulation. 2011;123(7):798-813.

4. Franchi F, Rollini F, Angiolillo DJ. Defining the link between chronic kidney disease, high platelet reactivity, and clinical outcomes in clopidogreltreated patients undergoing percutaneous coronary intervention. Circ Cardiovasc Interv. 2015;8(6):e002760.

5. Baber U, Farkouh ME, Arbel Y, Muntner P, Dangas G, Mack MJ, Hamza $\mathrm{TH}$, Mehran R, Fuster V. Comparative efficacy of coronary artery bypass surgery vs percutaneous coronary intervention in patients with diabetes and multivessel coronary artery disease with or without chronic kidney disease. Eur Heart J. 2016;37(46):3440-7.

6. Franchi F, James SK, Ghukasyan Lakic T, Budaj AJ, Cornel JH, Katus HA, Keltai M, Kontny F, Lewis BS, Storey RF, et al. Impact of diabetes mellitus and chronic kidney disease on cardiovascular outcomes and platelet P2Y12 receptor antagonist effects in patients with acute coronary syndromes: insights from the PLATO trial. J Am Heart Assoc. 2019;8(6):e011139.

7. Vranckx P, Valgimigli M, Juni P, Hamm C, Steg PG, Heg D, van Es GA, McFadden EP, Onuma Y, van Meijeren C, et al. Lancet. 2018;392:940.

8. Mehran R, Baber U, Sharma SK, Cohen DJ, Angiolillo DJ, Briguori C, Cha JY, Collier T, Dangas G, Dudek D, et al. Ticagrelor with or without aspirin in high-risk patients after PCI. N Engl J Med. 2019;381:2032.

9. Watanabe H, Domei T, Morimoto T, Natsuaki M, Shiomi H, Toyota T, Ohya M, Suwa S, Takagi K, Nanasato M, et al. Effect of 1-month dual antiplatelet therapy followed by clopidogrel vs 12-month dual antiplatelet therapy on cardiovascular and bleeding events in patients receiving $\mathrm{PCl}$ : the STOPDAPT-2 randomized clinical trial. JAMA. 2019;321(24):2414-27.

10. Hahn JY, Song YB, Oh JH, Chun WJ, Park YH, Jang WJ, Im ES, Jeong JO, Cho BR, Oh SK, et al. Effect of P2Y12 inhibitor monotherapy vs dual antiplatelet therapy on cardiovascular events in patients undergoing percutaneous coronary intervention: The SMART-CHOICE Randomized Clinical Trial. JAMA. 2019;321(24):2428-37.

11. Bruck K, Stel VS, Gambaro G, Hallan S, Volzke H, Arnlov J, Kastarinen M, Guessous I, Vinhas J, Stengel B, et al. CKD prevalence varies across the european general population. J Am Soc Nephrol. 2016;27(7):2135-47.

12. Geiss LS, Wang J, Cheng YJ, Thompson TJ, Barker L, Li Y, Albright AL, Gregg EW. Prevalence and incidence trends for diagnosed diabetes among adults aged 20 to 79 years, United States, 1980-2012. JAMA. 2014;312(12):1218-26.

13. Vranckx P, Valgimigli M, Windecker S, Steg PG, Hamm C, Juni P, GarciaGarcia HM, van Es GA, Serruys PW. Long-term ticagrelor monotherapy versus standard dual antiplatelet therapy followed by aspirin monotherapy in patients undergoing biolimus-eluting stent implantation: rationale and design of the GLOBAL LEADERS trial. Eurolntervention. 2016;12(10):1239-45.

14. Stevens LA, Coresh J, Greene T, Levey AS. Assessing kidney function-measured and estimated glomerular filtration rate. N Engl J Med. 2006;354(23):2473-83.

15. Costa F, van Klaveren D, James S, Heg D, Räber L, Feres F, Pilgrim T, Hong $\mathrm{M}-\mathrm{K}$, Kim H-S, Colombo A, et al. Derivation and validation of the predicting bleeding complications in patients undergoing stent implantation and subsequent dual antiplatelet therapy (PRECISE-DAPT) score: a pooled analysis of individual-patient datasets from clinical trials. The Lancet. 2017;389(10073):1025-34.

16. Vranckx P, Valgimigli M, Jüni $P$, Hamm C, Steg PG, Heg D, van Es GA, McFadden EP, Onuma Y, van Meijeren C, et al. Ticagrelor plus aspirin for 1 month, followed by ticagrelor monotherapy for 23 months vs aspirin plus clopidogrel or ticagrelor for 12 months, followed by aspirin monotherapy for 12 months after implantation of a drug-eluting stent: a multicentre, open-label, randomised superiority trial. The Lancet. 2018;392(10151):940-9.

17. Mehran R, Rao SV, Bhatt DL, Gibson CM, Caixeta A, Eikelboom J, Kaul S, Wiviott SD, Menon V, Nikolsky E, et al. Standardized bleeding definitions for cardiovascular clinical trials: a consensus report from the Bleeding Academic Research Consortium. Circulation. 2011;123(23):2736-47.

18. Cutlip DE, Windecker S, Mehran R, Boam A, Cohen DJ, van Es GA, Steg PG, Morel MA, Mauri L, Vranckx P, et al. Clinical end points in coronary stent trials: a case for standardized definitions. Circulation. 2007;115(17):2344-51.

19. Garcia-Garcia HM, McFadden EP, Farb A, Mehran R, Stone GW, Spertus J, Onuma Y, Morel MA, van Es GA, Zuckerman B, et al. Standardized end point definitions for coronary intervention trials: the academic research consortium-2 consensus document. Eur Heart J. 2018;39(23):2192-207.

20. Li G, Taljaard M, Van den Heuvel ER, Levine MA, Cook DJ, Wells GA, Devereaux PJ, Thabane L. An introduction to multiplicity issues in clinical trials: the what, why, when and how. Int J Epidemiol. 2017;46(2):746-55.

21. Best PJ, Lennon R, Ting HH, Bell MR, Rihal CS, Holmes DR, Berger PB. The impact of renal insufficiency on clinical outcomes in patients undergoing percutaneous coronary interventions. J Am Coll Cardiol. 2002;39(7):1113-9.

22. James S, Budaj A, Aylward P, Buck KK, Cannon CP, Cornel JH, Harrington RA, Horrow J, Katus H, Keltai M, et al. Ticagrelor versus clopidogrel in acute coronary syndromes in relation to renal function: results from the Platelet Inhibition and Patient Outcomes (PLATO) trial. Circulation. 2010;122(11):1056-67.

23. Du Y, Heidemann C, Schaffrath Rosario A, Buttery A, Paprott R, Neuhauser $H$, Riedel T, Icks A, Scheidt-Nave C. Changes in diabetes care indicators: findings from German National Health Interview and Examination Surveys 1997-1999 and 2008-2011. BMJ Open Diabetes Res Care. 2015;3(1):e000135.

24. Bramlage $P$, Lanzinger S, van Mark G, Hess E, Fahrner S, Heyer CHJ, Friebe M, Seufert J, Danne T, Holl RW. Patient and disease characteristics of type-2 diabetes patients with or without chronic kidney disease: an analysis of the German DPV and DIVE databases. Cardiovasc Diabetol. 2019;18(1):33. 
25. Palanca A, Castelblanco E, Betriu A, Perpinan H, Soldevila B, Valdivielso JM, Bermudez-Lopez M, Puig-Jove C, Puig-Domingo M, Groop PH, et al. Subclinical atherosclerosis burden predicts cardiovascular events in individuals with diabetes and chronic kidney disease. Cardiovasc Diabetol. 2019;18(1):93.

26. Bonaca MP, Bhatt DL, Cohen M, Steg PG, Storey RF, Jensen EC, Magnani G, Bansilal S, Fish MP, Im K, et al. Long-term use of ticagrelor in patients with prior myocardial infarction. N Engl J Med. 2015;372(19):1791-800.

27. Bhatt DL, Bonaca MP, Bansilal S, Angiolillo DJ, Cohen M, Storey RF, Im K, Murphy SA, Held P, Braunwald E, et al. Reduction in ischemic events with ticagrelor in diabetic patients with prior myocardial infarction in PEGASUS-TIMI 54. J Am Coll Cardiol. 2016;67(23):2732-40.

28. Angiolillo DJ, Baber U, Sartori S, Briguori C, Dangas G, Cohen DJ, Mehta SR, Gibson CM, Chandiramani R, Huber K, et al. Ticagrelor with or without aspirin in high-risk patients with diabetes mellitus undergoing percutaneous coronary intervention. J Am Coll Cardiol. 2020;75(19):2403-13.

29. Bhatt DL, Steg PG, Mehta SR, Leiter LA, Simon T, Fox K, Held C, Andersson $\mathrm{M}$, Himmelmann A, Ridderstråle W, et al. Ticagrelor in patients with diabetes and stable coronary artery disease with a history of previous percutaneous coronary intervention (THEMIS-PCI): a phase 3, placebocontrolled, randomised trial. The Lancet. 2019;394(10204):1169-80.

30. Steg PG, Bhatt DL, Simon T, Fox K, Mehta SR, Harrington RA, Held C, Andersson M, Himmelmann A, Ridderstrale W, et al. Ticagrelor in patients with stable coronary disease and diabetes. N Engl J Med. 2019;381(14):1309-20.

31. Baber U, Mehran R, Giustino G, Cohen DJ, Henry TD, Sartori S, Ariti C, Litherland C, Dangas G, Gibson CM, et al. Coronary thrombosis and major bleeding after PCI with drug-eluting stents: risk scores from PARIS. J Am Coll Cardiol. 2016;67(19):2224-34

\section{Publisher's Note}

Springer Nature remains neutral with regard to jurisdictional claims in published maps and institutional affiliations.
Ready to submit your research? Choose BMC and benefit from:

- fast, convenient online submission

- thorough peer review by experienced researchers in your field

- rapid publication on acceptance

- support for research data, including large and complex data types

- gold Open Access which fosters wider collaboration and increased citations

- maximum visibility for your research: over $100 \mathrm{M}$ website views per year

At BMC, research is always in progress.

Learn more biomedcentral.com/submissions 